\title{
Lithography-Free Fabrication of Silica Nanocylinders with Suspended Gold Nanorings for LSPR-Based Sensing
}

Thilsted, Anil Haraksingh; Pan, Jesper Yue; Wu, Kaiyu; Zor, Kinga; Rindzevicius, Tomas; Schmidt, Michael Stenbæk; Boisen, Anja

\section{Published in:}

Small

Link to article, DOI:

10.1002/smll.201602299

Publication date:

2016

Document Version

Peer reviewed version

Link back to DTU Orbit

Citation (APA):

Thilsted, A. H., Pan, J. Y., Wu, K., Zor, K., Rindzevicius, T., Schmidt, M. S., \& Boisen, A. (2016). LithographyFree Fabrication of Silica Nanocylinders with Suspended Gold Nanorings for LSPR-Based Sensing. Small, 12(48), 6745-6752. https://doi.org/10.1002/smll.201602299

\section{General rights}

Copyright and moral rights for the publications made accessible in the public portal are retained by the authors and/or other copyright owners and it is a condition of accessing publications that users recognise and abide by the legal requirements associated with these rights.

- Users may download and print one copy of any publication from the public portal for the purpose of private study or research.

- You may not further distribute the material or use it for any profit-making activity or commercial gain

- You may freely distribute the URL identifying the publication in the public portal 


\title{
Lithography-Free Fabrication of Silica Nanocylinders with Suspended Gold Nanorings for LSPR-Based Sensing
}

\author{
Anil Haraksingh Thilsted \\ Jesper Yue Pan \\ Kaiyu Wu \\ Kinga Zor \\ Tomas Rindzevicius \\ Michael Stenbæk Schmidt \\ Anja Boisen \\ Department of Micro- and Nanotechnology \\ The Technical University of Denmark \\ 2800 Kongens Lyngby, Denmark \\ Email: anja.boisen@nanotech.dtu.dk
}

$5 / 29 / 2017$ 


\begin{abstract}
Tunable plasmonic platforms are important for a variety of applications ranging from photovoltaics, LED's and optoelectronics, medical research and biosensors. In particular, development of label-free plasmonic biosensors is one of key research areas that utilize plasmonic nanostructures for detection of biologically relevant molecules at low concentrations. We have developed a costeffective, fast and lithography-free method to fabricate transparent fused silica nanocylinders. The technique allows tuning of nanocylinder height, width and density and can be scaled to large surface areas, such as 8 inch wafers. We demonstrate that gold coated nanocylinders support localized surface plasmon resonances (LSPR) from visible to NIR wavelengths. The plasmonic platform can be characterized as suspended gold nanorings and exhibit a sensitivity of $658 \mathrm{~nm} \mathrm{RIU}^{-1}$ with a figure-of-merit (FoM) of 10, comparable to other state-of-the-art LSPR sensing platforms that utilize more complex nanofabrication pathways. It was observed that the LSPR peak positions can be controlled by varying the morphology of the nanocylinders. We illustrate surface functionalization, biosensing and surface regeneration properties of the platform using thiols and detection of bovine serum albumin (BSA). The observed LSPR shifts for 11mercaptoundecanoic acid and BSA was $12 \mathrm{~nm}$ and $26 \mathrm{~nm}$, respectively.
\end{abstract}

\title{
1 Introduction
}

Noble metal nanostructures display interesting optical properties that are particularly relevant for biosensing applications [1]. The nanoparticle optical response to an incident electromagnetic field is known as the localized surface plasmon resonance (LSPR) and is due to collective resonant oscillations of the conduction electrons. LSPRs can be excited by light, which leads to a strong enhancement of the local field and exhibits prominent light absorption and scattering [2,3]. The nanoparticle optical response is usually dominated by dipolar modes and the LSPR peak position depends strongly on nanostructure geometry and refractive index (RI) of the surrounding medium [4]. Typically, refractive index and molecular sensing using LSPRs are carried out using chemically synthesized solutions of silver or gold colloids. Measurements of the LSPR response, as a function of the RI change have either been carried out on single or an ensemble of particles, with varying degrees of performance [5]. Advances in nanofabrication techniques have allowed for more precise control of nanostructure morphology, significantly improving optical properties and resulting in advances in sensing applications, commonly by means of measuring a shift in the LSPR peak position [6]. Recently, a new class of extremely sensitive biosensing platforms based on two-dimensional porous gold nanorod arrays or hyperbolic metamaterials that can reach sensitivities of $30,000 \mathrm{~nm}$ per refractive index unit (RIU) 
have been reported [7, 8]. Even though both gold nanorod and hyperbolic metamaterials exhibit high sensitivities, the former is feasible only in the infrared spectral region while the latter involves complex fabrication procedures which can limit their use in routine point-of-care (POC) applications [9]. In fact, most plasmonic platform fabrication techniques often involve either top-down processes such as electron-beam lithography $[10,11]$, an expensive and time-consuming process that can only pattern small areas, or advanced bottom-up techniques such as block copolymer [12] or nanosphere lithography [13, 14].

Extensive work has been carried out both experimentally and theoretically to characterise and tune the optical properties of various nanoparticles $[15,16]$ and nanocavity $[17,18]$ based plasmonic structures. Specifically, metallic rings or toris have shown promise as highly tunable plasmonic structures [19, 20] with a wellunderstood analytical and finite-difference time-domain model for the electric field distribution [21, 22]. Gold nanorings exhibit significant field enhancement useful for highly sensitive LSPR based detection[23, 24, 25]. Additionally, it has been shown that the sensitivity of plasmonic structures can be increased significantly by simply raising the metallic structures above the substrate. For structures at a near proximity to the substrate, a large portion of the field will be in the substrate where it is insensitive to RI changes. The effect of suspending the structures above the substrate was explored by Dmitriev et al.[26] as well as Otte et al.[27] who showed significant performance increase. It is therefore important to combine the sensitivity and large tunability range of plasmonic metal nanorings with the sensitivity of metal structures suspended above the substrate. The structures are preferably achieved by a straightforward and fast fabrication method.

In contrast to nanosphere and e-beam lithography, maskless RIE forms nanostructures in a single and fast fabrication step. When combined with a subsequent metallization step, the process has been utilized for producing wafer-scale silver and and gold coated silicon nanopillar arrays for surface-enhanced Raman scattering (SERS) applications [28, 29]. While maskless nanostructuring in silicon is well understood, the lack of optical transparency, limited chemical resistance and insufficient biocompatibility leave much to be desired for sensing applications [30]. It is therefore desirable to extend the method for fabricating plasmonic structures to optically transparent materials. A method for achieving nanostructures in fused silica using lithography-free RIE was initially proposed by Zeze et al. [31] and further developed for a variety of other nanostructures by Lilienthal et al. [30] and Hein et al. [32]. Prior to this work, optimization of the nanocylinder geometry along with precise control of the height, width and density of the nanocylinders has not been reported. In particular, we propose that the maskless RIE of fused silica could be used to realize suspended gold nanoring configurations.

In order to address the drawbacks of complicated and expensive nanofabrication steps that are generally employed to produce plasmonic biosensing platforms, we have developed a fast, cost-effective and simple fabrication method that yields waferscale fused silica nanocylinder structures. Importantly, we demonstrate control of the geometry of fused silica nanocylinders achieved by only adjusting RIE process parameters. The deposited gold layer results in isolated metal rings at the tops of the nanocylinders serving as plasmon resonance structures suspended above the substrate. We show that the optical properties of suspended gold nanorings can be controlled by tuning the dimensions and density of the nanocylinders through the RIE process parameters. Furthermore, we evaluate the LSPR sensing characteristics of the platform by measuring the RI sensitivity, FoM and recording molecular binding 
events. Since the proposed configuration can be achieved on wafer-scale, the practical realization of a sensor is achievable for a range of applications and is suitable for POC devices.

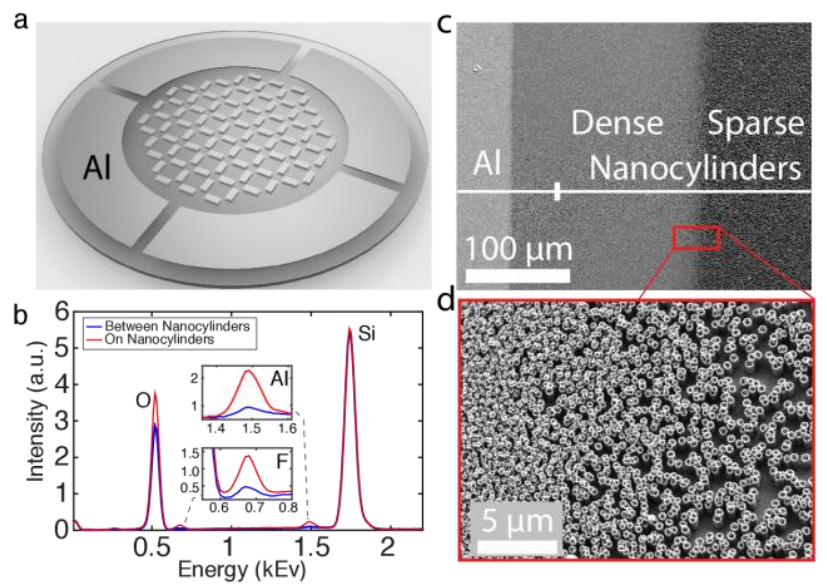

Figure 1: (a) The aluminum pattern used on the 4 inch silica wafer, with the outer ring ensuring electrostatic clamping in the etch chamber and the inner rectangle array to ensure homogeneous nanocylinder density.(b) EDX measurements show the presence of aluminum and fluorine, and heightened signal on the sites of the nanocylinders. (c,d) SEM images showing how aluminum sputtering causes nanocylinders to form. The nanocylinders are dense near the aluminum areas, but from $200 \mu \mathrm{m}$ and out, they become less dense and with a homogeneous distribution.

\section{Results and Discussion}

\subsection{Fabrication of Nanocylinders}

Nanocylinders were fabricated on the surface of fused silica wafers via a one-step self-masking RIE process. Sulfur hexafluoride $\left(\mathrm{SF}_{6}\right)$ and dioxygen $\left(\mathrm{O}_{2}\right)$ gas were injected into a vacuum chamber, and ionised to form a plasma using a radio frequency oscillating electric field. The ions of the plasma were accelerated in an additional electric field to impact the surface of the substrate and etch material away. A predeposited aluminum layer on the fused silica resulted in sputtered residues that functioned as nanoscale masks. These nanoscale masks induce local differences in etching selectivity, thus forming the nanostructures. However, the formation of nanocylinders due to these nanoscale masks is not fully understood [31]. An illustration of the predeposited aluminum pattern and SEM images of the resulting nanocylinders is illustrated in Figure 1. 
The flow rate of gases, the coil power used to generate the plasma, the platen power used to accelerate the ions, the pressure in the plasma chamber and the temperature of the substrate must all be carefully balanced in order to form the desired structures. Additionally, details such as the configuration of the RIE tool, the wafer cooling method, and size of the substrate and chamber conditions before etching may have significant affects on the geometry of the nanostructures. The etching process is strongly dependent on the initial chamber conditions and therefore, a 3 min $\mathrm{O}_{2}$ chamber cleaning was carried out before each etch. As the formation of the nanocylinders requires sputtering off the aluminum pattern, the morphology was dependent on the distance to the mask. Large variations in the density of the nanocylinders were observed if the fused silica wafer did not have sufficient coverage of aluminum. In order to ensure homogeneiety across the wafer, an aluminum pattern was designed to ensure a maximum distance of $2.75 \mathrm{~mm}$ to the aluminum layer from the glass regions. Nanocylinders were found to be much denser within $200 \mu \mathrm{m}$ from the aluminum layer, but thereafter became more sparse and had a homogeneous density. Additionally, as the etching process is dependent on the specific tool used, some modification of the etching parameters is required if transferred to other instruments. An etch process was developed that served as the starting point for etch parameter variation, listed in Table 1.
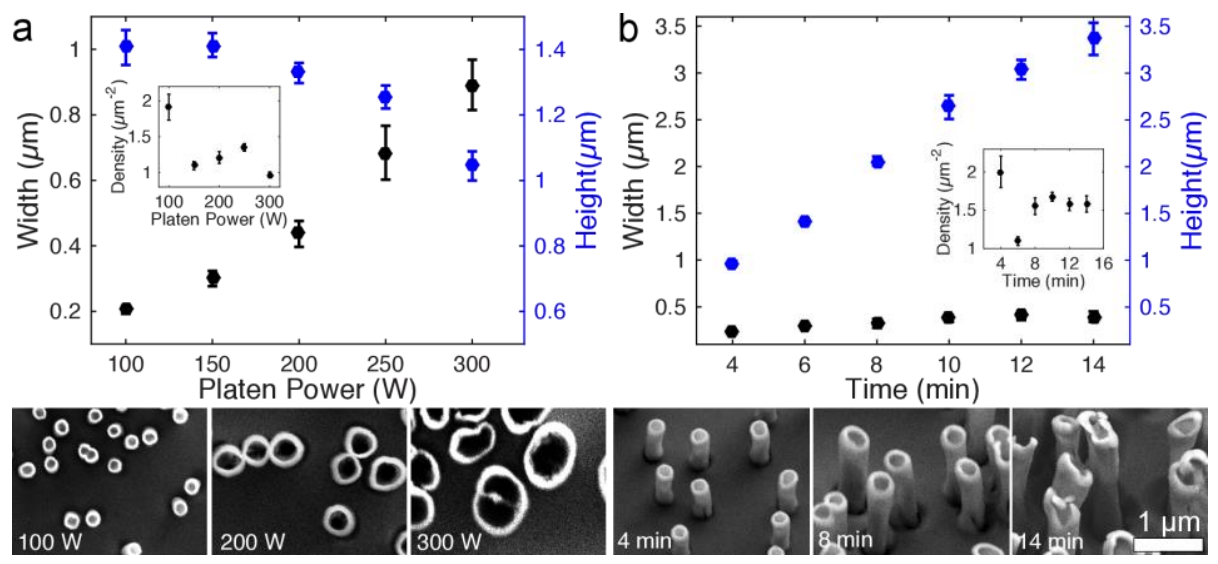

Figure 2: Control of the morphology of the nanocylinders by varying the platen power and time of the etching process. (a) Increasing the platen power increases the diameter of the nanocylinders and a slight decrease in the height is observed. The density decreases as the available space decreases with wider nanocylinders. (b) Increasing the etching time results in taller nanocylinders, with almost no change in the nanocylinder width. The density decreases initially with increasing etching time but stabilises at $\sim 1.6 \mu \mathrm{m}^{-2}$. Errorbars show the standard deviation resulting from measurements of dimensions, taken at different locations across the entire wafer using SEM imaging. 


\begin{tabular}{|l|r|}
\hline $\mathrm{SF}_{6}$ flow & $150 \mathrm{sccm}$ \\
\hline $\mathrm{O}_{2}$ flow & $30 \mathrm{sccm}$ \\
\hline Pressure & $40 \mathrm{mTorm}$ \\
\hline Platen power & $120 \mathrm{~W}$ \\
\hline Coil power & $800 \mathrm{~W}$ \\
\hline Chuck temperature & $20^{\circ} \mathrm{C}$ \\
\hline Etch time & $6 \mathrm{~min}$ \\
\hline
\end{tabular}

\section{Table 1: Standard Nanocylinder Etching Process}

This etch process resulted in nanocylinders with a height of $1410 \pm 36 \mathrm{~nm}$, a width of $300 \pm 23 \mathrm{~nm}$ and a density of $1.10 \pm 0.06 \mu \mathrm{m}^{-2}$. The nanocylinder geometry was measured across the entire wafer and a distributed aluminum mask was used as an effective way to produce homogeneous results across a large area.

It is possible to tune the height and width of the nanocylinders by varying the etching parameters. The dimensions and densities of the nanocylinder as a function of platen power and etch time are plotted in Figure 2. Increasing platen power from 100 $W$ to $300 \mathrm{~W}$ resulted in nanocylinders with a diameter increasing from $0.20 \mu \mathrm{m}$ to $0.89 \mu \mathrm{m}$ with only a decrease in height from $1.41 \mu \mathrm{m}$ to $1.04 \mu \mathrm{m}$. The decrease in height was most likely due to the walls of the nanocylinders being etched down with a more physical etching process. For nanocylinders above $\sim 800 \mathrm{~nm}$ in width, structures were observed to form inside the hollow cores - possibly nanocylinders within nanocylinders. The density was observed to decrease from $1.91 \mu \mathrm{m}^{-2}$ to $0.97 \mu \mathrm{m}^{-2}$, as platen power increased from $100 \mathrm{~W}$ to $300 \mathrm{~W}$.

Increasing the etching time from $4 \mathrm{~min}$ to $14 \mathrm{~min}$ resulted in an increase in height from $0.96 \mu \mathrm{m}$ to $3.36 \mu \mathrm{m}$ with almost no change in width. However, for nanocylinders with a height greater than $\sim 2.5 \mu \mathrm{m}$, tearing and buckling were observed, especially at the base of the nanocylinders - possibly due to thinning of sidewalls and subsequent mechanical failure. As with increasing platen power, the density decreased from $2.00 \mu \mathrm{m}^{-2}$ and stabilized at $\sim 1.5 \mu \mathrm{m}^{-2}$ for etch times above 8 $\min$. By combining certain platen powers and etch times, a desired width, height and density for the nanocylinders can be achieved despite the stochastic nature of the RIE process.

E-beam deposition resulted in metallic rings along the rim of the nanocylinders and a continuous thin film between the nanocylinders, shown in Figure 3. At the base of the nanocylinders, cavities in the gold film arose due to the masking effect of the nanocylinders during gold deposition. The cavity size was estimated to be slightly larger than the width of the nanocylinders. The gold rings' outer diameters were the same as the width of the nanocylinders, whereas the thicknesses of the gold ring walls were $90.7 \mathrm{~nm}$. 

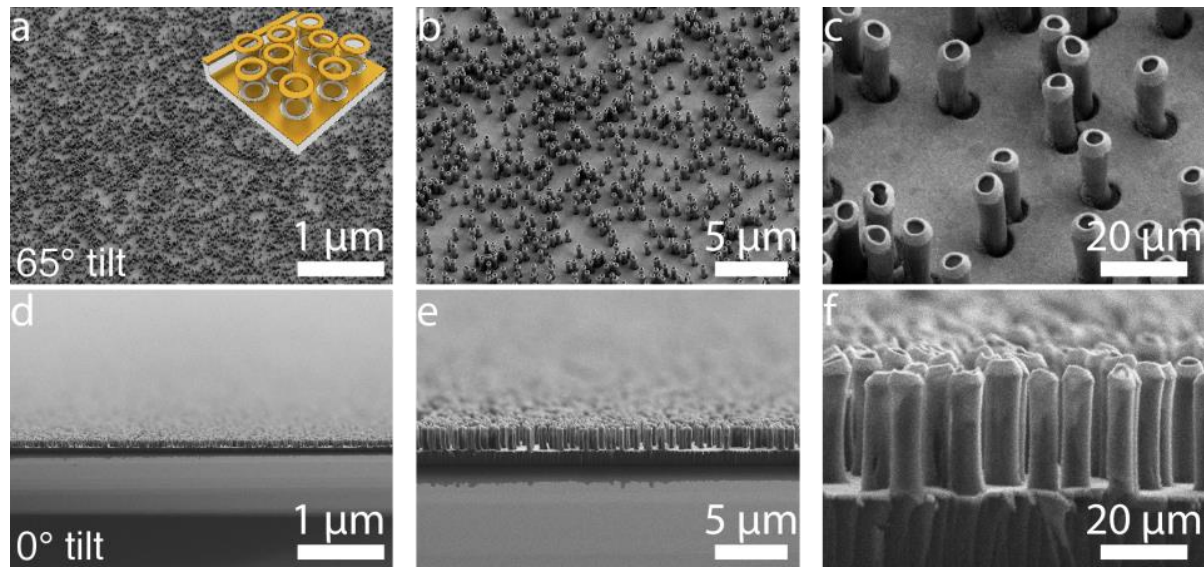

Figure 3: SEM images showing nanocylinders with suspended gold rings and with a continuous gold film between them. The insert in (a) shows a sketch of the silica nanocylinders after gold deposition with suspended gold rings and a gold film between the nanocylinders. The SEM images are shown for $(a, b, c)$ at $65^{\circ}$ tilt and at $(\mathrm{d}, \mathrm{e}, \mathrm{f}) 0^{\circ}$ tilt at different magnifications, showing a homogeneous density, height and width of the suspended gold rings.

\subsection{Optical Properties}

A qualitative understanding of the resonance modes were investigated using 3D FEM simulations using mean geometric values of fabricated nanocylinders: $300 \mathrm{~nm}$ diameter, $1413 \mathrm{~nm}$ height and a gold layer thickness of $50 \mathrm{~nm}$. The electric field distribution at different wavelengths were observed and the predicted absorption cross-section was compared with measured extinction spectra, ash shown in Figure 4. The model showed three clear modes observed between wavelengths of $600 \mathrm{~nm}$ and $1100 \mathrm{~nm}$ : the asymmetric mode at $\sim 630 \mathrm{~nm}$ in which there is a strong coupling between the inner and outer part of the gold torus wall; the symmetric mode at $\sim 1090$ $\mathrm{nm}$ in which the particle exhibits a dipolar resonance; and a hybrid mode of the symmetric and antisymmetric mode at $\sim 810 \mathrm{~nm}$. These resonance modes were likewise observed in the extinction spectra. 

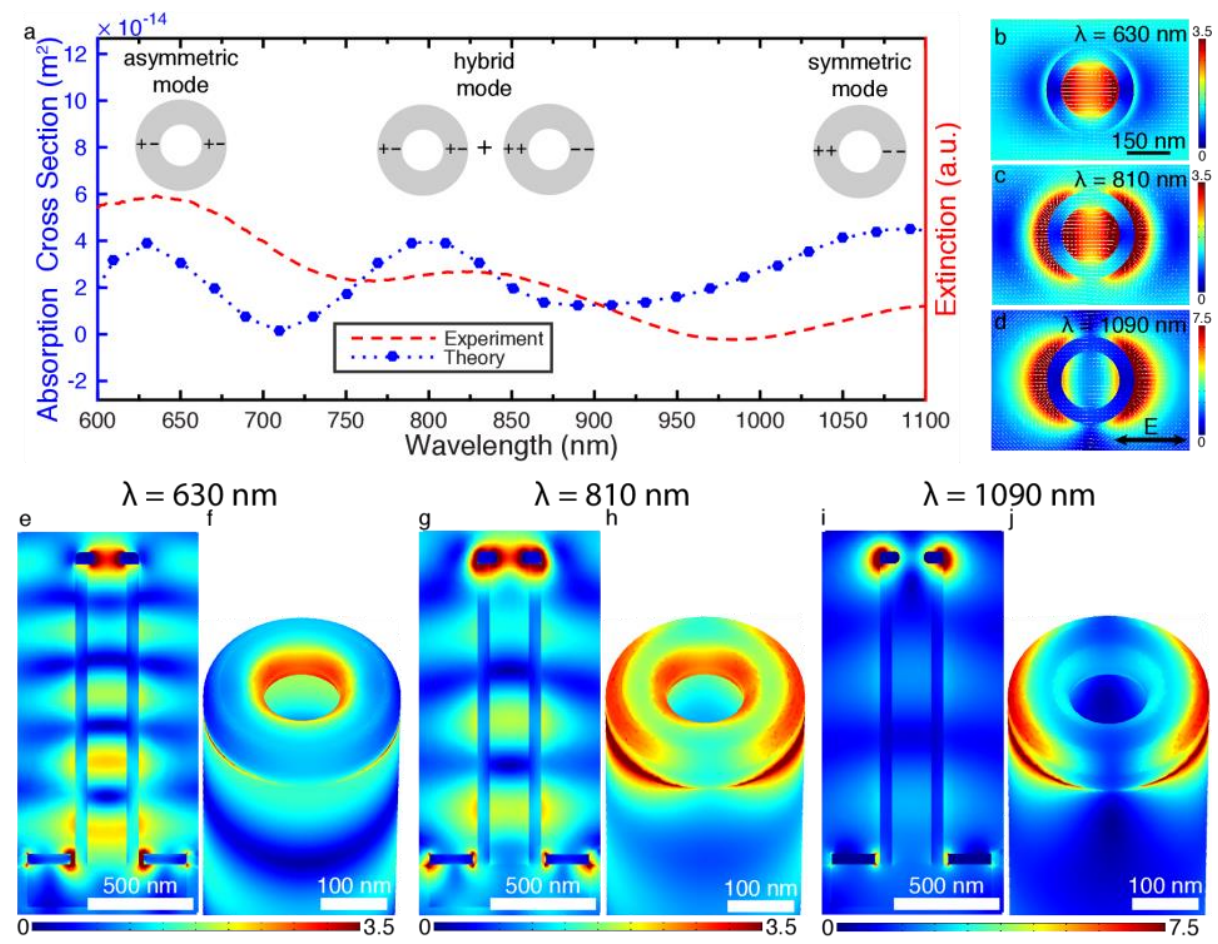

Figure 4: (a) A comparison of absorption cross section calculated from a simulation with measurements of the extinction spectrum showing three distinct peaks between $600 \mathrm{~nm}$ and $1100 \mathrm{~nm}$ corresponding to three resonance modes. (b) Top-down view of the electric field distribution of the asymmetric mode and (c) the hybrid mode and the (d) symmetric mode. (e,f) The cross-sectional view of the electric field and (b) tilted view of the electric field at the surface of the gold nanoring for the asymmetric mode at $\lambda=630 \mathrm{~nm}$, for the (c,d) hybrid mode at $\lambda=810 \mathrm{~nm}$ and the (e,f) symmetric mode at $\lambda=1090 \mathrm{~nm}$. The scale bars show the electric field enhancement.

The model does not take into account the inter-nanocylinder electromagnetic coupling and stochastic spread in morphology and scattering. Despite this shortcoming, the model agrees qualitatively with experiments.

The ability to tune the morphology of nanocylinders in a predictable fashion allows for tuning of the LSPR peaks. Extinction spectra were measured for nanocylinders varying from $205 \mathrm{~nm}$ to $684 \mathrm{~nm}$ in diameter, with a $50 \mathrm{~nm}$ gold layer thickness. Note, that the change in nanocylinder diameter accompanied a change in density and height as described above. The resulting extinction spectra for varying nanocylinder diameters are shown in Figure 5. 

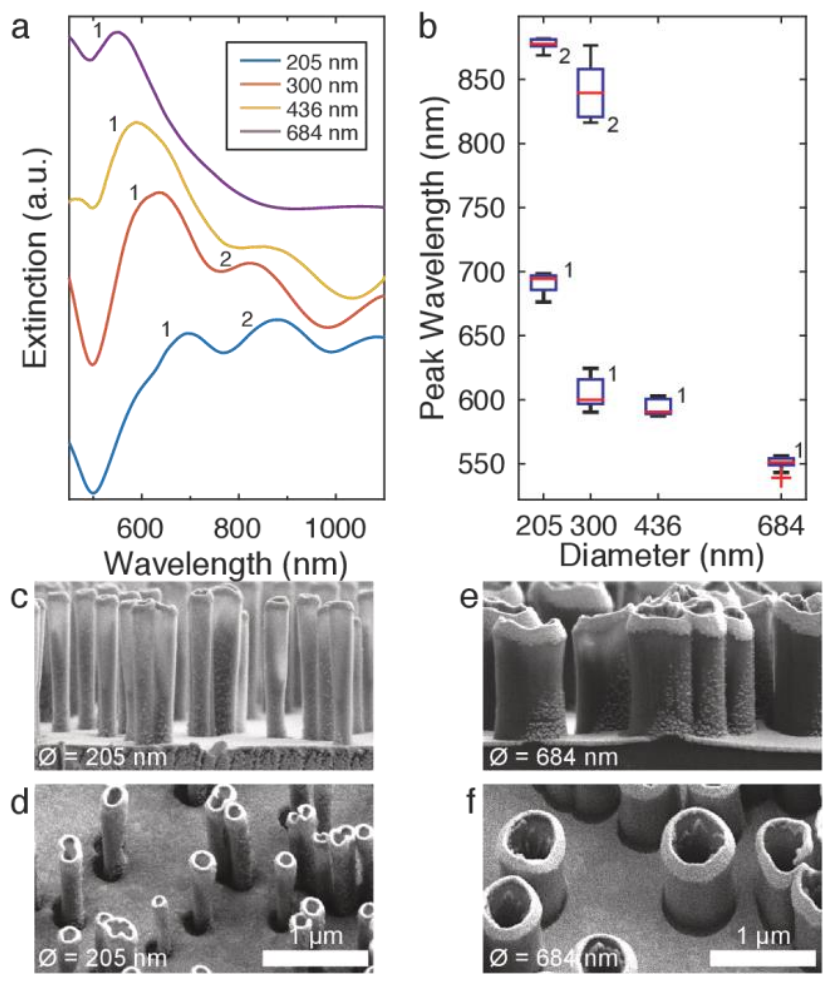

Figure 5: (a) Representative extinction spectra of nanocylinders with varying diameters. Three LSPR peaks between $500 \mathrm{~nm}$ and $1100 \mathrm{~nm}$ were observed as well as the gold intraband transition antipeak at 500 $n m$. (b) Increasing nanocylinder diameters resulted in a blue-shift of the asymmetric mode from $691 \mathrm{~nm}$ to $550 \mathrm{~nm}$ as well as a $877 \mathrm{~nm}$ to 793 $\mathrm{nm}$ blue-shift for the hybrid mode. Boxplots are based on 10 measurements at different wafer locations. Errorbars extend to data limits that are not considered outliers. (c,d) SEM micrographs of gold capped nanocylinders of small diameters and $(e, f)$ larger diameters.

Extinction spectra show three distinct peaks between $500 \mathrm{~nm}$ and $1100 \mathrm{~nm}$ with the exception of the nanocylinders with a diameter of $684 \mathrm{~nm}$. The peak positions of the LSPR modes were found to blue-shift from $691 \mathrm{~nm}$ to $550 \mathrm{~nm}$ for the asymmetric mode and from $877 \mathrm{~nm}$ to $793 \mathrm{~nm}$ for the hybrid mode. Although not directly calculated, as the LSPR peak extended beyond the measurement capabilities of the instrument, the measurements suggested a red-shift of the symmetric mode with increasing nanocylinder diameter.

The blue-shift of the asymmetric and hybrid LSPR peak modes was attributed to possibly retardation effects in intraparticle electromagnetic interaction, as described by Aizpurua et al. [19]. Additionally, the inferred red shift of the third mode was attributed to the larger gold ring size for wider nanocylinders. 
Varying the nanocylinder height is another way of tuning the LSPR peak position. Extinction spectra were measured for nanocylinders with heights between $0.96 \mu \mathrm{m}$ and $2.63 \mu \mathrm{m}$ in height. The extinction spectra are shown in Figure 6.
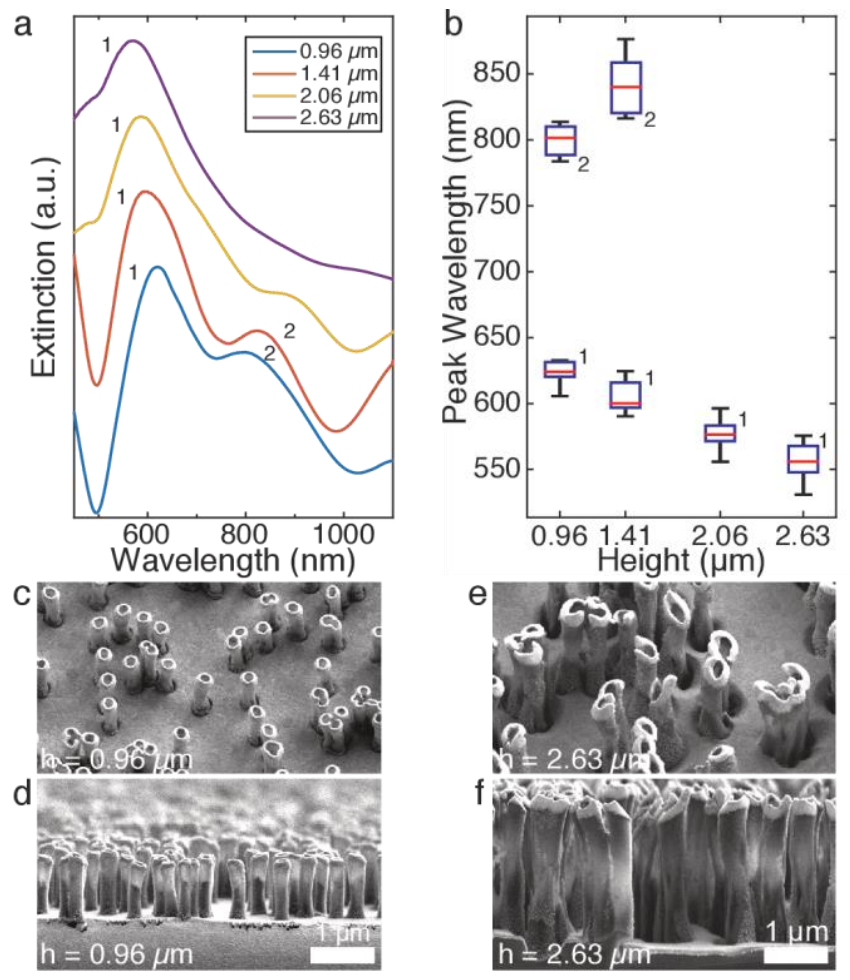

Figure 6: (a) Representative extinction spectra of nanocylinders with varying heights. Three LSPR peaks between $500 \mathrm{~nm}$ and $1100 \mathrm{~nm}$ were observed. (b) Increasing the nanocylinder height resulted in a linear blue-shift of the asymmetric mode from wavelength $624 \mathrm{~nm}$ to $556 \mathrm{~nm}$. The hybrid mode was observed to red-shift as well as become weaker for taller nanocylinders. Boxplots are based on 10 measurements at different locations across a wafer. (c,d) SEM micrographs of shorter nanocylinders and (e,f) taller nanocylinders with $50 \mathrm{~nm}$ gold caps.

Three LSPR peaks were identified between wavelength $500 \mathrm{~nm}$ and $1100 \mathrm{~nm}$. A linear blue-shift from $624 \mathrm{~nm}$ to $556 \mathrm{~nm}$ was observed for the asymmetric LSPR peak. In contrast to this, a red-shift from $800 \mathrm{~nm}$ to $841 \mathrm{~nm}$ was observed as the nanocylinder height increased from $0.96 \mu \mathrm{m}$ to $1.41 \mu \mathrm{m}$. The hybird peak became more red-shifted and weaker as the height increased further to $2.06 \mu \mathrm{m}$ and disappeared completely for taller nanocylinders.

The ability to fabricate nanocylinders of a certain height and width allows for tuning the LSPR peaks for the asymmetric, hybrid and symmetric modes in the 
visible to near infrared regions. In addition, varying the gold thickness allows allows for LSPR peak tuning (see Supplementary Information).

\subsection{Refractive Index Sensing}

Measurements of bulk RI sensitivity and FoM was carried out using sucrose solutions of varying concentrations. Representative extinction spectra and peak wavelength shifts for varying RI solutions are shown in Figure 7.

Peak shift showed a strong linear response to increased cladding refractive index. Nanocylinders with a height of $962 \mathrm{~nm}$, widths of $300 \mathrm{~nm}$ and $204 \mathrm{~nm}$ showed a RI

sensitivity of $474 \mathrm{~nm} / \mathrm{RIU}, 595 \mathrm{~nm} \mathrm{RIU^{-1 }}$ and $550 \mathrm{~nm} R \mathrm{RI}^{-1}$, respectively. The peak width at half prominence was found to increase for higher RI, indicating peak broadening. Using the average peak width at half prominence, the FoM was calculated to be 4.01, 5.18 and 5.57, respectively. The nanocylinders with the greatest sensitivity, $685 \mathrm{nmRIU}^{-1}$, were nanocylinders with a height of $2060 \mathrm{~nm}$. Additionally, nanocylinders with this height exhibit a peak width at half prominence of $69.3 \mathrm{~nm}$, resulting in a FoM of 9.87. The symmetric mode at the longest wavelength may have even higher sensitivity as indicated by previous work with metal nanorings [25].

Surface RI sensing was verified using two commonly used thiols: 11mercaptoundecanoic acid (MUA) (10 $\mathrm{mM}$ in ethanol) and cystamine hydrochloride (Cys) (10 $\mathrm{mM}$ in water). The formation of a self-assembled monolayer of thiols resulted in shifts in the LSPR peak position. Structures modified with Cys were further incubated in bovine serum albumin (BSA) (1.5 $\mu M$ in a phosphate buffered saline (PBS) solution), chosen as a model protein. The binding of the protein to the positively charged thiol layer could be reversed by a 15 minute incubation in Tris buffer ( $\mathrm{pH}$ 9.9). Extinction spectra were measured in dry states. The extinction spectra and the calculated peak shifts for the Cys covalent binding to the gold surface as well as the electrostatic interaction between the BSA and the Cys are shown in Figure 7. 

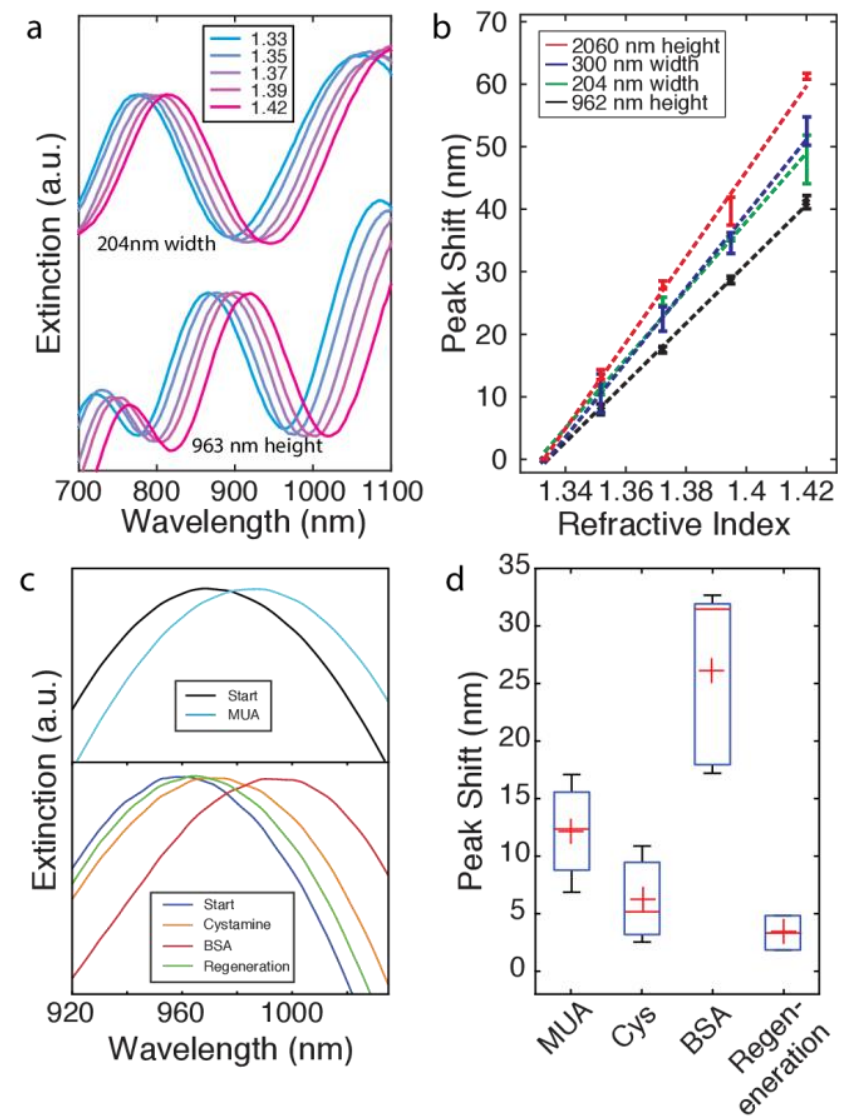

Figure 7: (a) Representative extinction spectra of nanocylinders immersed in sucrose solutions with varying refractive indices. The redshifting spectra are shown for nanocylinders with a width of $204 \mathrm{~nm}$ and nanocylinders with a height of $963 \mathrm{~nm}$. (b) A plot of the LSPR peak wavelength as a function of the refractive index. Dashed lines show a linear regression. Three measurements were taken for each nanocylinder geometry and refractive index. A linear increase of peak shift is evident and nanocylinders with a height of $2060 \mathrm{~nm}$ have the greatest sensitivity of $685 \mathrm{~nm} \mathrm{RIU}^{-1}$. (c) Representative spectra showing a peak shift as a result of MUA and Cys binding to the gold surfaces, as well as BSA interaction with Cys, before and after regeneration. (d) Peak shifts due to the Cys binding and BSA interaction with $\mathrm{Cys}$ before and after regeneration. Three measurements were carried out between at least two substrates. Crosses denote the mean value.

Extinction spectra showing a significant red-shift of $12.2 \pm 4.4 \mathrm{~nm}$ for MUA binding to the surface and $6.18 \pm 4.27 \mathrm{~nm}$ for Cys binding. BSA binding via 
electrostatic interaction to the Cys covered gold surfaces resulted in a peak shift of $26.3 \pm 7.8 \mathrm{~nm}$. Regeneration of the surface showed that it was possible to detect the removal of BSA using the peak shift, dropping the peak shift back to $3.3 \pm 2.2 \mathrm{~nm}$. These peaks shifts are on level with results obtained by comparable substrates fabricated using lithographic methods $[33,25]$.

\section{Conclusion}

In this paper, we report a fabrication method using RIE to achieve large area, high density nanocylinders that are easily tunable in both width and height. The fabrication process is straightforward, cost-effective, fast and compatible with high volume manufacturing process flows. A gold coating gold achieved with e-beam deposition results in suspended gold nanorings, exhibiting plasmonic properties. Using optical transmission, several prominent LSPR extinction peaks were measured in the VISNIR range. The maximum RI sensitivity of $685 \mathrm{~nm} \mathrm{RIU}^{-1}$ was found with gold nanocylinders with a height of $2060 \mathrm{~nm}$, a diameter of $324 \mathrm{~nm}$ and a density of $1.55 \mathrm{\mu m}^{-2}$. The peak width at half prominence was $69.3 \mathrm{~nm}$, resulting in a FoM of 9.87. Surface binding events were characterized, showing a peak shift of $12.2 \pm 4.4 \mathrm{~nm}$ for MUA, $6.18 \pm 4.27 \mathrm{~nm}$ for Cys and 26.25 $\pm 7.8 \mathrm{~nm}$ for BSA. These findings suggest that suspended gold rings on transparent fused silica nanocylinders fabricated using RIE is a strong candidate for a highly tunable, sensitive, low-cost sensor for LSPR bio/chemical detection using LSPR.

\section{Acknowledgement}

Thanks to Rodrigo Pimental for his assistance with 3D printing chip holders. This work was conducted by the NAPLAS project, funded by the Danish Council for Independent Research and the European Research Council under the European Union's Seventh Framework Programme (FP7/2007-2013) / ERC grant agreement $\mathrm{n}^{\mathrm{o}}$ 320535.

\section{Experimental Section}

\subsection{Fabrication and Characterization}

The nanocylinders were fabricated using 4 inch double side polished fused silica wafers with a $500 \mu \mathrm{m}$ thickness ${ }^{1}$. The aluminum mask ${ }^{2}$ was achieved using e-beam evaporation through an aluminum shadowmask. The wafers were then placed in a reactive ion etcher tool ${ }^{3}$. The gold film was deposited using the e-beam evaporator. Nanostructures were characterized using a scanning electron microscope ${ }^{4}$.

\footnotetext{
${ }^{1}$ Innergie Technologies, Inc.

${ }^{2}$ Alcatel SCM 600 E-beam and sputtering deposition system

${ }^{3}$ Advanced Oxide Etcher: STS MESC Multiplex ICP

${ }^{4}$ Zeiss Supra VP 40 SEM
} 


\subsection{Simulation and Optical Characterization}

Simulation of the plasmonic structures and prediction of the LSPR peak position was carried out using finite-element modelling in $\mathrm{COMSOL}^{5}$ using the radio-frequency (RF) module. A simplified model was constructed consisting of a single nanocylinder with a gold ring placed at the top rim of the nanocylinder. The top of the substrate surrounding the nanocylinder was also covered using gold film. The boundaries of the model were enclosed in perfectly matched layers (PML). Refractive index (RI) of the gold layer was obtained from Johnson et al. [34] and the RI of fused silica was obtained from Malitson et al. [35]. The electric field distribution was calculated after introducing a planar wave incident to the substrate surface with varying wavelength.

Optical characterization of the gold coated nanocylinders was carried out using a transmission spectrophotometer ${ }^{6}$. A $2 \times 2 \mathrm{~mm}$ area of the wafer was selected and measurements were carried out from $\lambda=400 \mathrm{~nm}$ to $\lambda=1100 \mathrm{~nm}$. LSPR peak positions were calculated using by first approximating a position using MatLab's findpeaks function. Varying data window sizes were defined around this peak guess and each data crop was fitted to a binomial (see Supplementary Information). The maximum position of the binomial fit determined the peak position.

\subsection{Bulk RI Sensing}

Bulk RI sensing was characterized using sucrose solutions ${ }^{7}$ at varying concentrations. The LSPR substrates were placed in a cuvette and a light blocker was used so that all light passed through the solution as well as the substrate (see Supplementary Information). Sucrose solutions were prepared from $0^{\circ} \mathrm{Bx}$ to $50^{\circ} \mathrm{Bx}$, corresponding to a RI shift from 1.333 to $1.4201^{8}$.

\subsection{Surface Sensing}

Cysteamine hydrochloride (Cys), 11-Mercaptoundecanoic acid (MUA), bovine serum albumin (BSA), phosphate buffered saline (PBS), TrizmaÂßbase, sodium hydroxide, were purchased form were purchased from Sigma-Aldrich Corporation (St. Louis, MO, USA). 99\% ethanol and absolute ethanol were from Kemetyl A/S (Køge, Denmark). In all cases, after the removal of the functionalization or incubation solution, the structures were rinsed three times with water and dried prior measurement. All aqueous solutions were prepared in ultrapure water, obtained from a Milli-Q® ${ }^{\circledR}$ water purification system ${ }^{9}$.

\section{References}

[1] Katherine A Willets and Richard P Van Duyne. Localized surface plasmon resonance spectroscopy and sensing. Annual review of physical chemistry, 58:267-97, jan 2007.

\footnotetext{
${ }^{5}$ COMSOL Inc.

${ }^{6}$ Shimadzu, UV-1800 UV-Vis Spectrophotometer

${ }^{7}$ Sigma Aldrich

${ }^{8}$ BrixChart

${ }^{9}$ Millipore Corporation, Billerica, MA, USA
} 
[2] M. Faraday. AuNP117-The Bakerian Lecture: Experimental Relations of Gold (and Other Metals) to Light. Philosophical Transactions of the Royal Society of London, 147(0):145-181, 1857.

[3] Gustav Mie. Beiträge zur Optik trüber Medien, speziell kolloidaler Metallösungen. Annalen der Physik, 330(3):377-445, 1908.

[4] Craig F. Bohren and Donald R. Huffman. Absorption and Scattering of Light by Small Particles. John Wiley \& Sons, 2008.

[5] Kathryn M. Mayer and Jason H. Hafner. Localized surface plasmon resonance sensors. Chemical Reviews, 111(6):3828-3857, 2011.

[6] Jeffrey N Anker, W Paige Hall, Olga Lyandres, Nilam C Shah, Jing Zhao, and Richard P Van Duyne. Biosensing with plasmonic nanosensors. Nature materials, 7(June):442-453, 2008.

[7] a V Kabashin, P Evans, S Pastkovsky, W Hendren, G a Wurtz, R Atkinson, R Pollard, V a Podolskiy, and a V Zayats. Plasmonic nanorod metamaterials for biosensing. Nature materials, 8(11):867-871, 2009.

[8] Kandammathe Valiyaveedu Sreekanth, Yunus Alapan, Mohamed ElKabbash, Efe Ilker, Michael Hinczewski, Umut A. Gurkan, Antonio De Luca, and Giuseppe Strangi. Extreme sensitivity biosensing platform based on hyperbolic metamaterials. Nature Materials, (March):4-11, 2016.

[9] Frank B Myers and Luke P Lee. Innovations in optical microfluidic technologies for point-of-care diagnostics. Lab on a chip, 8(12):2015-2031, 2008.

[10] Niels Verellen, Pol Van Dorpe, Chengjun Huang, Kristof Lodewijks, Guy A E Vandenbosch, Liesbet Lagae, and Victor V Moshchalkov. Plasmon Line Shaping Using Nanocrosses for High Sensitivity.pdf.

NanoLetters, pages 391-397, 2011.

[11] Sarah Kim, Jin Mi Jung, Dae Geun Choi, Hee Tae Jung, and Seung Man Yang. Patterned arrays of au rings for localized surface plasmon resonance. Langmuir, 22(13):7109-7112, 2006.

[12] Dong Ok Shin, Jong-Ryul Jeong, Tae Hee Han, Chong Min Koo, Hye-Jeong Park, Yong Taik Lim, and Sang Ouk Kim. A plasmonic biosensor array by block copolymer lithography. Journal of Materials Chemistry, 20(34):7241, aug 2010.

[13] Traci R Jensen, Michelle Duval Malinsky, Christy L Haynes, and Richard P Van Duyne. Nanosphere lithography: tunable localized surface plasmon resonance spectra of silver nanoparticles. Journal of Physical Chemistry B, 104(45):10549-10556, 2000.

[14] Erin M. Hicks, Xiaoyu Zhang, Shengli Zou, Olga Lyandres, Kenneth G. Spears, George C. Schatz, and Richard P. Van Duyne. Plasmonic properties of film over nanowell surfaces fabricated by nanosphere lithography. Journal of Physical Chemistry B, 109(47):22351-22358, 2005.

[15] Amanda J Haes, Shengli Zou, George C Schatz, and Richard P Van Duyne. Nanoscale Optical Biosensor : Short Range Distance Dependence of the Localized Surface Plasmon Resonance of Noble Metal Nanoparticles. Journal of Physical Chemistry B, pages 6961-6968, 2004.

[16] E Hutter and J H Fendler. Exploitation of localized surface plasmon resonance. Advanced Materials (Weinheim, Germany), 16(19):1685-1706, 2004. 
[17] Antoine Lesuffleur, Hyungsoon Im, Nathan C. Lindquist, and SangHyun Oh. Periodic nanohole arrays with shape-enhanced plasmon resonance as real-time biosensors. Applied Physics Letters, 90(24):243110, 2007.

[18] Magnus P Jonsson, Andreas B Dahlin, Peter Jönsson, and Fredrik Höök. Nanoplasmonic biosensing with focus on short-range ordered nanoholes in thin metal films. Biointerphases, 3(3):FD30-D40, 2008.

[19] J Aizpurua, P Hanarp, D S Sutherland, M Käll, Garnett W Bryant, and F J García de Abajo. Optical properties of gold nanorings. Physical review letters, 90(5):057401, 2003.

[20] Peter Nordlander. The Ring: A leitmotif in plasmonics. ACS Nano, 3(3):488-492, 2009.

[21] Chizuko M Dutta, Tamer a Ali, Daniel W Brandl, Tae-Ho Park, and Peter Nordlander. Plasmonic properties of a metallic torus. The Journal of chemical physics, 129(8):084706, 2008.

[22] Feng Hao, Peter Nordlander, Mathew T. Burnett, and Stefan A. Maier. Enhanced tunability and linewidth sharpening of plasmon resonances in hybridized metallic ring/disk nanocavities. Physical Review B, 76(24):245417, 2007.

[23] Francesco De Angelis, Mario Malerba, Maddalena Patrini, Ermanno Miele, Gobind Das, Andrea Toma, Remo Proietti Zaccaria, and Enzo Di Fabrizio. 3D Hollow Nanostructures as Building Blocks for Multifunctional Plasmonics. Nano Letters, 13(8):3553-3558, 2013.

[24] Chengjun Huang, Jian Ye, Shuo Wang, Tim Stakenborg, and Liesbet Lagae. Gold nanoring as a sensitive plasmonic biosensor for on-chip DNA detection. Applied Physics Letters, 100(17):17-21, 2012.

[25] Elin M. Larsson, Joan Alegret, Mikael Käll, and Duncan S. Sutherland. Sensing characteristics of NIR localized surface plasmon resonances in gold nanorings for application as ultrasensitive biosensors. Nano Letters, 7(5):1256-1263, 2007.

[26] Alexandre Dmitriev, Carl Hägglund, Si Chen, Hans Fredriksson, Tavakol Pakizeh, Mikael Käll, and Duncan S. Sutherland. Enhanced nanoplasmonic optical sensors with reduced substrate effect. Nano Letters, 8(11):3893-3898, 2008.

[27] Marinus a. Otte, M. Carmen Estévez, Laura G. Carrascosa, Ana B. González-Guerrero, Laura M. Lechuga, and Borja Sepúlveda. Improved biosensing capability with novel suspended nanodisks. Journal of Physical Chemistry C, 115(13):5344-5351, 2011.

[28] Michael Stenbaek Schmidt, Jörg Hübner, and Anja Boisen. Large area fabrication of leaning silicon nanopillars for surface enhanced Raman spectroscopy. Advanced materials (Deerfield Beach, Fla.), 24(10):OP11-8, mar 2012.

[29] Kaiyu Wu, Tomas Rindzevicius, Michael Stenbæk Schmidt, Klaus Bo Mogensen, Sanshui Xiao, and Anja Boisen. Plasmon resonances of Ag capped Si nanopillars fabricated using mask-less lithography. Optics Express, 23(10):12965, 2015.

[30] Katharina Lilienthal, Mike Stubenrauch, Michael Fischer, and Andreas Schober. Fused silica â€ glass grass': fabrication and utilization. Journal of Micromechanics and Microengineering, 20(2):025017, feb 2010. 
[31] D. a. Zeze, D. C. Cox, B. L. Weiss, and S. R. P. Silva. Lithographyfree high aspect ratio submicron quartz columns by reactive ion etching.

Applied Physics Letters, 84(8):1362, 2004.

[32] Eric Hein, Dennis Fox, and Henning Fouckhardt. Glass surface modification by lithography-free reactive ion etching in an Ar/CF4-plasma for controlled diffuse optical scattering. Surface and Coatings Technology,

205:S419-S424, 2011.

[33] Yang Shen, Jianhua Zhou, Tianran Liu, Yuting Tao, Ruibin Jiang, Mingxuan Liu, Guohui Xiao, Jinhao Zhu, Zhang-Kai Zhou, Xuehua Wang, Chongjun Jin, and Jianfang Wang. Plasmonic gold mushroom arrays with refractive index sensing figures of merit approaching the theoretical limit. Nature communications, 4:2381, 2013.

[34] P. B. Johnson and R. W. Christry. Optical Constants of the Noble Metals. Physical Review B, 6(12):4370-4379, 1972.

[35] I. H. Malitson. Interspecimen Comparison of the Refractive Index of Fused Silica. Journal of the Optical Society of America, 55(10):1205, 1965. 